\title{
Golf Green Slope Estimation Using a Cross Laser Structured Light System and an Accelerometer
}

\author{
Duy Duong Pham*, Quoc Khanh Dang* and Young Soo Suh ${ }^{\dagger}$
}

\begin{abstract}
In this paper, we propose a method combining an accelerometer with a cross structured light system to estimate the golf green slope. The cross-line laser provides two laser planes whose functions are computed with respect to the camera coordinate frame using a least square optimization. By capturing the projections of the cross-line laser on the golf slope in a static pose using a camera, two 3D curves' functions are approximated as high order polynomials corresponding to the camera coordinate frame. Curves' functions are then expressed in the world coordinate frame utilizing a rotation matrix that is estimated based on the accelerometer's output. The curves provide some important information of the green such as the height and the slope's angle. The curves estimation accuracy is verified via some experiments which use OptiTrack camera system as a ground-truth reference.
\end{abstract}

Keywords: Golf slope estimation, Accelerometer, Structured light

\section{Introduction}

The laser structured light system has been used for several decades in many areas since it provides high accuracy 3D measurement. It is applied for welding detection [1], 3D depth map construction [2], polyhedral surfaces location and orientation [3], and 3D object identification [4]. The laser structured light system consists of a laser (line or cross type) together with a camera. The laser projects a line (or cross line) on the scene and the camera captures images containing the projection. For each laser point in each image, the 3D position can be computed if the relative pose between the laser and camera is known [5].

In this paper, we apply a laser structure light system to a golf green slope angle estimation problem. In the golf putting, it is important to correctly estimate the green slope angle. However, it is not always easy to read the green slope correctly $[6,7]$, which is especially true for novice players. Inclinometers (placed on the green) are often used to aid the slope angle estimation [8]. Although the inclinometer gives an accurate slope angle, it can give only one point slope angle. We propose a new method of estimating the green slope angles combining a cross laser structured light and an accelerometer. The accelerometer is used to estimate the attitude of the camera, which is needed to transform the 3D position data (which is described in terms of the camera coordinate system) into the slope angle. Since smartphones contain an accelerometer sensor unit, the proposed system can be constructed by simply attaching a laser to a smartphone.

$\dagger$ Corresponding Author: Dept. of Electrical Engineering, University of Ulsan, Korea. (yssuh@ulsan.ac.kr)

* Dept. of Electrical Engineering, University of Ulsan, Korea (\{duyduongd2, khanhdq8689\}@gmail.com)

Received: April 8, 2015; Accepted: September 30, 2015
This paper is organized as below. Section 2 shows problem formulations and solutions. In Section 3, we introduce a camera and laser plane calibration problem and in Section 4 we present a slope estimation algorithm. The experimental results are given in Section 5 and conclusions are presented in Section 6.

\section{Problem Formulation}

During the putting time in the golf, one of the most important things is to predict the motion of ball near the hole. The trajectory of the ball depends heavily on the slope but sometimes it is not easy to correctly estimate the slope angle. In this paper, the slope angle is estimated using a camera, a cross laser and a three axis accelerometer unit.

There are four coordinate frames: world, accelerometer, camera and laser coordinate frames. The $z$ axis of the world coordinate system coincides with the local gravitational (upward) direction. The $x$ direction is not important and can be chosen arbitrarily. The three axes of the accelerometer coordinate system coincide with three axes of an accelerometer unit. The $z$ axis of the camera coordinate system is defined as shown in Fig. 1, which is the standard way to define the camera coordinate system. One line of the cross line laser is assumed to lie on the $z x$ plane (plane 1) of the laser coordinate system and the other line is assumed to lie on the $y z$ plane (plane 2) of the laser coordinate system. Here the cross laser lines are assumed to be perpendicular.

We need to know the relative attitude between the accelerometer unit and the camera as well as the relative pose between the laser and the camera. Let rotation matrix $R_{c}^{a} \in R^{3 \times 3}$ represent the rotation relationship from the 


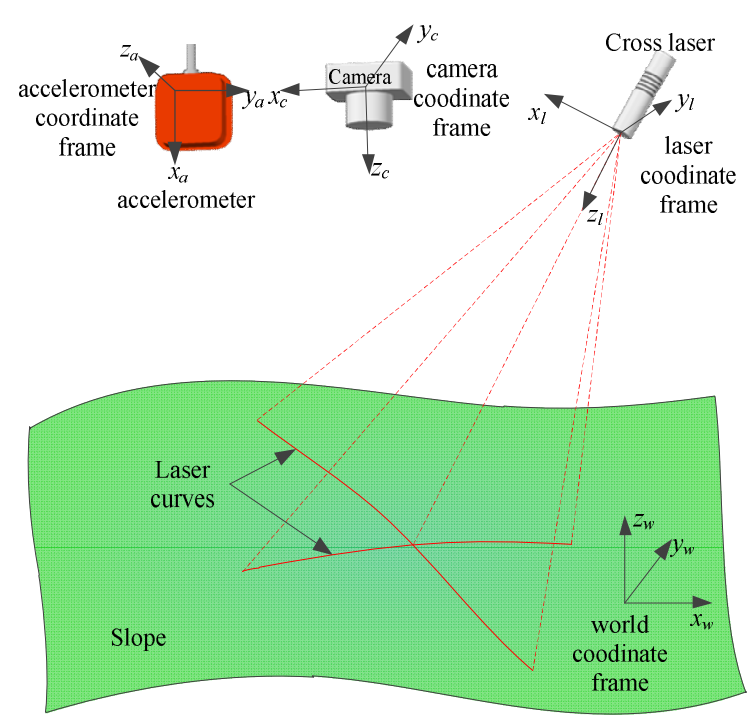

Fig. 1. System overview

camera coordinate system to the accelerometer coordinate system. For a vector $p \in R^{3}$, we have the following relationship:

$$
[p]_{a}=R_{c}^{a}[p]_{c}+\left[t_{c}\right]_{a}
$$

where $[p]_{a}\left([p]_{c}\right)$ represents that the vector $p$ is expressed in the accelerometer (camera) coordinate system. Similarly we use the notation $[p]_{l}$ and $[p]_{w}$ for a vector in the laser and world coordinate systems, respectively. The translation vector $\left[t_{c}\right]_{a}$ is not required in this paper since only the attitude is essential. The relative pose between the laser and camera can be expressed using two plane equations with respect to the camera coordinate system [9] (see Fig. 2):

$$
\begin{aligned}
& \left(n_{1}, r\right)=d_{1}, \\
& \left(n_{2}, r\right)=d_{2}
\end{aligned}
$$

where $(a, b)$ represents the inner product of vectors $a \in R^{3}$ and $b \in R^{3} . n_{1} \in R^{3}$ and $n_{2} \in R^{3}$ are unit normal vectors of two laser planes, respectively.

The required calibration parameters are $R_{c}^{a}, n_{1}, d_{1}, n_{2}$ and $d_{2}$, which are computed in Section 3.1 and 3.2. The camera intrinsic parameters are computed using the method in Chapter 11 of [10].

\section{Sensors Calibrations}

In this section, the calibration parameters are computed, where a checkerboard with known checker size is used.

\subsection{Computation of $n_{1}, d_{1}, n_{2}$ and $d_{2}$}

A checkerboard is placed on a flat table. Without losing

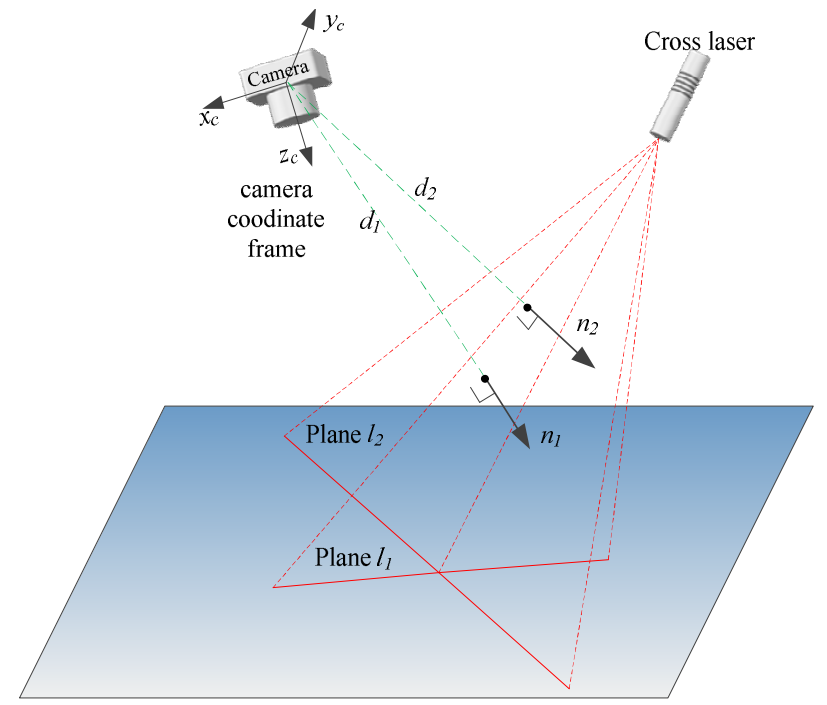

Fig. 2. Relationship between laser and camera

generality, it is assumed that the checkerboard is on the $x y$ plane of the world coordinate system. With arbitrarily chosen position and attitude, two pictures are taken with and without cross laser lines alternately. After that, the camera position and attitude are changed and two pictures are taken again by the same way. This step is repeated $N_{c a l}$ times and an index $i\left(1 \leq i \leq N_{c a l}\right)$ is used to denote the step number.

Two line equations are derived for two cross laser lines in the checkerboard (see Fig. 4 for one laser line). Let two line equations (line equations are derived using points in the normalized image plane) are given by

$$
\begin{aligned}
& A_{1, i} x+B_{1, i} y+C_{1, i}=0 \\
& A_{2, i} x+B_{2, i} y+C_{2, i}=0
\end{aligned}
$$

where $\left[A_{j, i} B_{j, i} C_{j, i}\right]^{T}(j=1$ or 2$)$ is the unit vector and the index $i\left(1 \leq i \leq N_{c a l}\right)$ represents that the line equation corresponding to the $i-t h$ step image.

Consider the first line equation in (3). The origin of the camera coordinate frame and points on the first line equation form a plane as shown in Fig. 3 (see plane $c_{1}$ ). The plane $c_{1}$ equation is given by:

$$
\left(n_{c_{1, i}}, r\right)=d_{c_{1, i}}
$$

where

$$
n_{c_{1, i}}=\left[\begin{array}{c}
A_{1, i} \\
B_{1, i} \\
C_{1, i}
\end{array}\right], \quad d_{c_{1, i}}=0 .
$$

In (4), the plane $c_{1}$ is expressed in the camera coordinate system. Note that there are three planes in Fig. 


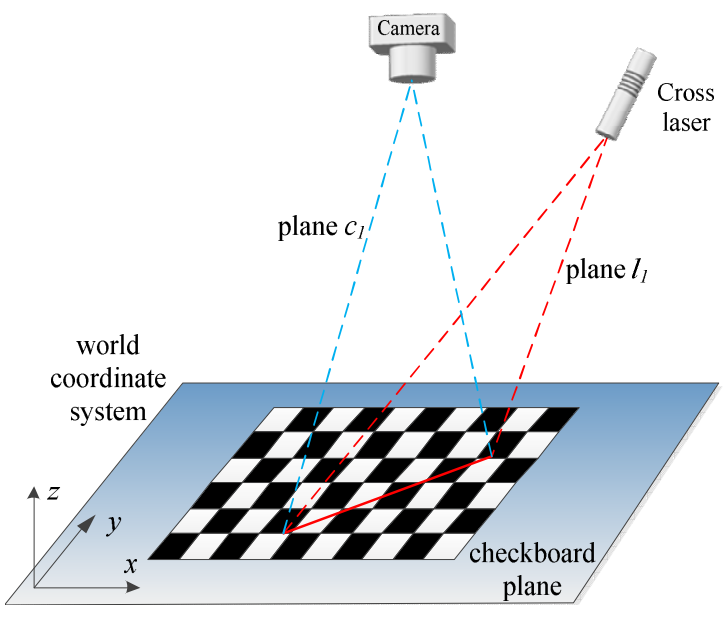

Fig. 3. Three planes: camera, laser, checkerboard planes

3: plane $c_{1}$, checkerboard plane ( $x y$ plane of the world coordinate system) and laser plane $l_{1}$. The laser line on the checkerboard is the intersection of these three planes. Using this fact, we are going to derive the plane $l_{1}$ equation $\left(n_{1}\right.$ and $\left.d_{1}\right)$ in the camera coordinate system. To do that, we first derive the checkerboard plane equation.

From the checkerboard image (with known checker size), we can compute the relative pose $R_{w}^{c} \in R^{3 \times 3}$ and $\left[t_{w}\right]_{c} \in R^{3}$ between the camera and the world coordinate systems using the method in [11] so that the following is satisfied for a vector $p \in R^{3}$ :

$$
[p]_{c}=R_{w}^{c}[p]_{w}+\left[t_{w}\right]_{c}
$$

The checkerboard plane equation (which is assumed to be on the $x y$ plane of the world coordinate system) in the world coordinate system is given by:

$$
\left(n_{\text {board }, w},[r]_{w}\right)=d_{\text {board }, w}
$$

where

$$
n_{\text {board }, w}=\left[\begin{array}{l}
0 \\
0 \\
1
\end{array}\right], \quad d_{\text {board }, w}=0 .
$$

Combining (5) and (6), the checkerboard plane equation in the camera coordinate system is given by:

$$
\left(n_{\text {board }, c},[r]_{c}\right)=d_{\text {board }, c}
$$

where

$$
\begin{gathered}
n_{\text {board }, c}=R_{w}^{c} n_{\text {board }, w}, \\
d_{\text {board }, c}=\left(n_{\text {board }, w},\left(R_{w}^{c}\right)^{\prime}\left[t_{w}\right]_{c}\right) .
\end{gathered}
$$

Now we are going to derive the laser line equation, which is the intersection of three planes in Fig. 3. We use $(p, n)$ line representation (see 4.2.2 in [9]), where a space point $r$ is on the line if the following is satisfied:

$$
r \times p=n
$$

where $\left[p^{\prime}, n^{\prime}\right]^{\prime} \in R^{6 \times 1}$ is the unit norm vector. Since the intersection line between plane $c_{1, i}$ and checkerboard plane is the same as the intersection line between plane $l_{1}$ and checkerboard, the following is derived using (4.72) in [9]:

$$
\begin{gathered}
N\left(\left[\begin{array}{c}
n_{c_{1, i}} \times n_{\text {board }, c} \\
d_{\text {board }, c} n_{c_{1, i}}-d_{c_{1, i}} n_{\text {board }, c}
\end{array}\right]\right) \\
\text { subject to }\left\|n_{1}\right\|=1 .
\end{gathered}
$$

In (9), $N(a)$ denote the normalized vector of a vector a. Note that the unknown parameters in (9) are $n_{1}$ and $d_{1}$. Equation (9) can be written in the following form:

$$
b_{1, i}=N\left(M_{1, i} x_{1}\right)
$$

where

$$
\begin{aligned}
& b_{1, i}=N\left(\left[\begin{array}{c}
n_{c_{1, i}} \times n_{\text {board }, c} \\
d_{\text {board }, c} n_{c_{1, i}}-d_{c_{1, i}} n_{\text {board }, c}
\end{array}\right]\right) \in R^{6}, \\
& M_{1, i}=\left[\begin{array}{cc}
{\left[n_{c_{1, i}} \times\right]} & 0 \\
-d_{c_{1, i}} I & n_{c_{1, i}}
\end{array}\right] \in R^{6 \times 4}, \\
& x_{1}=\left[\begin{array}{l}
n_{1} \\
d_{1}
\end{array}\right] \in R^{4 \times 1},\left\|n_{1}\right\|=1 .
\end{aligned}
$$

In (10), $[a \times]$ denotes the $3 \times 3$ matrix form of the cross product so that $a \times b=[a \times] b$. Since we have $N_{c a l}$ set of pictures, there are $N_{c a l}$ equations. Finding $x_{1}$ from $N_{c a l}$ equations can be written as the following optimization problem:

$$
\begin{aligned}
& \min _{\alpha_{i}, x}\left\|M_{1, i} x_{1}-\alpha_{1, i} b_{1, i}\right\| \\
& \text { subject to }\left\|C x_{1}\right\|=1
\end{aligned}
$$

where $C=\left[\begin{array}{ll}I_{3} & 0_{3 \times N_{\text {cal }}}\end{array}\right]$. If we define $\bar{x}_{1}$ as:

$$
\bar{x}_{1}=\left[\begin{array}{c}
x_{1} \\
\alpha_{1,1} \\
\vdots \\
\alpha_{1, N}
\end{array}\right] \in R^{\left(4+N_{c a l}\right) \times 1}
$$

(11) can be written as: 


$$
\begin{aligned}
& {\left[\begin{array}{ccccc}
M_{1,1} & -b_{1,1} & 0 & \ldots & 0 \\
M_{1,2} & 0 & -b_{1,2} & \ldots & 0 \\
\vdots & \vdots & \vdots & \vdots & \vdots \\
M_{1, N_{c a l}} & 0 & 0 & \ldots & -b_{1, N_{c a l}}
\end{array}\right] \bar{x}_{1}=0} \\
& \text { subject to } \|\left[\begin{array}{ccc}
C & \left.0_{3 \times\left(N_{c a l}+1\right)}\right] \bar{x}_{1} \|=1 .
\end{array}\right.
\end{aligned}
$$

The solution to (12) is given in A5.4.2 of [12]. Thus we can compute the plane $l_{1}$ equation in the camera coordinate system. Using the exactly same method, we can compute the plane $l_{2}$ equation in the camera coordinate system as well.

For an arbitrary image point $\left(x_{a}, y_{a}\right)$ given on the laser plane $l_{1}$, the corresponding $3 \mathrm{D}$ point in the camera coordinate frame is computed as

$$
s_{a}\left[\begin{array}{c}
x_{a} \\
y_{a} \\
1
\end{array}\right]
$$

where $s_{a}$ is a scaling factor computed based on the following:

$$
\left(n_{1}, s_{a}\left[\begin{array}{c}
x_{a} \\
y_{a} \\
1
\end{array}\right]\right)=d_{1} \Rightarrow s_{a}=\frac{d_{1}}{\left(n_{1},\left[\begin{array}{c}
x_{a} \\
y_{a} \\
1
\end{array}\right]\right)}
$$

\subsection{Rotation matrix between the accelerometer and the camera coordinate system}

The rotation matrix between the accelerometer and camera coordinate systems can be computed using the method proposed in [13]. The rotation can be estimated by having both sensors observe the vertical direction in several poses. In detail, the accelerometer measures the gravitational force while the camera captures vertical landmarks. We note that the system is static while taking data so the accelerometer only measures the gravitational force.

\section{Slope Estimation}

In this section, we propose a method to detect the golf slope using a cross-line laser system. The intersections of the laser planes and the golf slope create two curves as can be seen from Fig. 5a. From the calibration step in the Section 3, all 3D points of two laser planes are known in the camera coordinate frame if their coordinates in the camera images are defined. Based on that fact, the intersections of two laser planes and the golf slope can be approximated as two high order polynomials with respect to the camera coordinate frame. These curves are then expressed in the world coordinate frame by utilizing an accelerometer. The purpose of the accelerometer is to measure the gravitational force in order to estimate the relative pose between the system and the world coordinate frame. Since the camera images contain two laser stripes, our first step is to distinguish each 2D stripe in the images and later estimate the curves in $3 \mathrm{D}$ with respect to the world coordinate frame.

\subsection{Curves distinction}

Since the relative position of the camera and the laser is fixed, the intersection of two laser planes is also fixed with respect to the camera coordinate frame. Thus this intersection line is fixed in the image and can be used to distinguish two intersection stripes of laser planes and the golf slope.

Assume that $Q_{1}$ and $Q_{2}$ are two points in two laser planes' intersection line $L_{1}$, and their coordinates in the image coordinate frame are $Q_{1 c}=\left[\begin{array}{lll}x_{1} & y_{1} & 1\end{array}\right]^{T}, \quad Q_{2 c}=$ $\left[\begin{array}{lll}x_{2} & y_{2} & 1\end{array}\right]^{T}$ (see Fig. 5a).

The equation of the laser planes' intersection line in the image coordinate frame can be identified by $Q_{1 c}$ and $Q_{2 c}$ as follow:

$$
\frac{x-x_{1}}{x_{2}-x_{1}}=\frac{y-y_{1}}{y_{2}-y_{1}} \rightarrow y=a_{1} x+b_{1} \text {. }
$$

In Fig. 5b, two laser curves are separated by line $L_{1}$ and $L_{2}$. $L_{1}$ is the image of the intersection line between laser planes with the line equation given in (14). In each image, we can scan a bright point $\left(x_{0}, y_{0}\right)$ that belongs to the $L_{1}$ line function and satisfies $I\left(x_{0}, y_{0}\right)>\delta_{I}$, where $I\left(x_{i}, y_{i}\right)$ indicates the intensity of a point $\left(x_{i}, y_{i}\right)$ and $\delta_{I}$ is a chosen intensity threshold. However, due to the blurring of the image and the width of the laser line, there are a number of points satisfy above constraints. Therefore, the coordinate of intersection point $\left(\mathrm{x}_{0}, \mathrm{y}_{0}\right)$ between two curves in $L_{1}$ can be approximated by:

$$
x_{0}=\frac{\sum_{i=1}^{k} x_{i}}{k} \quad y_{0}=\frac{\sum_{i=1}^{k} y_{i}}{k}
$$

where $k$ is number of point $\left(x_{i}, y_{i}\right)$ in $L_{1}$. For simplicity, $L_{2}$ is a vertical line with the line function $x=x_{0}$ in the image. With these definitions, two lines $y=a_{1} x+b_{1}$ and $x=x_{0}$ split the image into four parts (see Fig. 5b). All laser points which belong to the part one and part three must be in the curve 1 , and the rest must be in the curve 2 . In other words, the condition of a point $\left(x_{j}, y_{j}\right)$ in

- curve 1 is:

$$
\left(y_{j}-\left(a_{1} x_{j}+b_{1}\right)\right)\left(x_{j}-x_{0}\right)<0,
$$




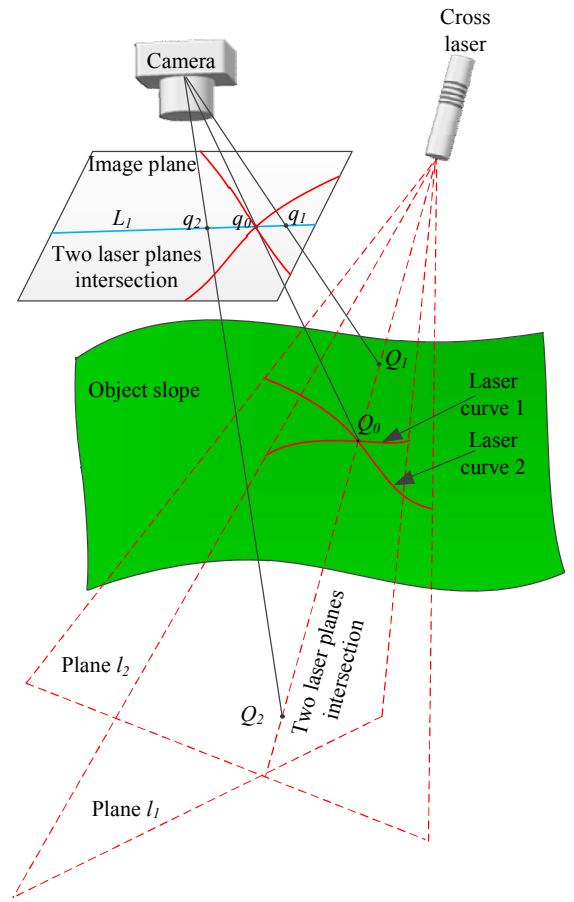

(a)

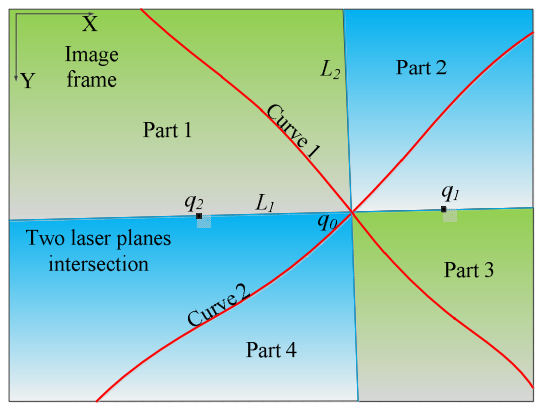

(b)

Fig. 5. (a) Intersection line (b) Separated curves

- curve 2 is:

$$
\left(y_{j}-\left(a_{1} x_{j}+b_{1}\right)\right)\left(x_{j}-x_{0}\right)>0 \text {. }
$$

An easiest way to define laser points in the image is to subtract two images which are captured in sequence so that the laser is turned on in the first frame while in the second frame the laser is off. By subtracting two images, an image that contains only laser stripes is obtained. This image is then converted from RGB format to Gray format in order to define the laser point coordinates which have higher intensities than the background. Conditions (16) and (17) are then used to classify point coordinates belonging to the first or the second curve.

\subsection{Curves estimation}

After obtaining a set of points belonging to the first and second curve, respectively, two basic curves are created based on representative points in each column in the image. An illustration for this process is described in Fig. 6 where a gray image of the laser line is constructed. A set of bright point coordinates collected from the gray image are subpixelled based on following weighting method [14]: in each $x_{k}$-th image column, $y_{k}$ is computed by:

$$
y_{k}=\frac{\sum_{j=1}^{n} y_{j} I\left(x_{k}, y_{j}\right)}{\sum_{j=1}^{n} I\left(x_{k}, y_{j}\right)}
$$

where $I\left(x_{k}, y_{j}\right)$ is the intensity of a pixel $\left(x_{k}, y_{j}\right), I$ is larger than a chosen intensity threshold and $n$ is the number laser points included in $x_{k}$-th image column. The basic curve formed from sub-pixelled points is then approximated to a high order polynomial in order to get a smooth curve function in the image frame.

Based on calibration steps the estimated curve function now can be expressed in the camera coordinate frame. Our next destination is to describe estimated curves with respect to the world coordinate frame as our goal is to estimate the golf slope in real situation. The procedure of golf slope estimation is briefly sketched out in Fig. 7 where

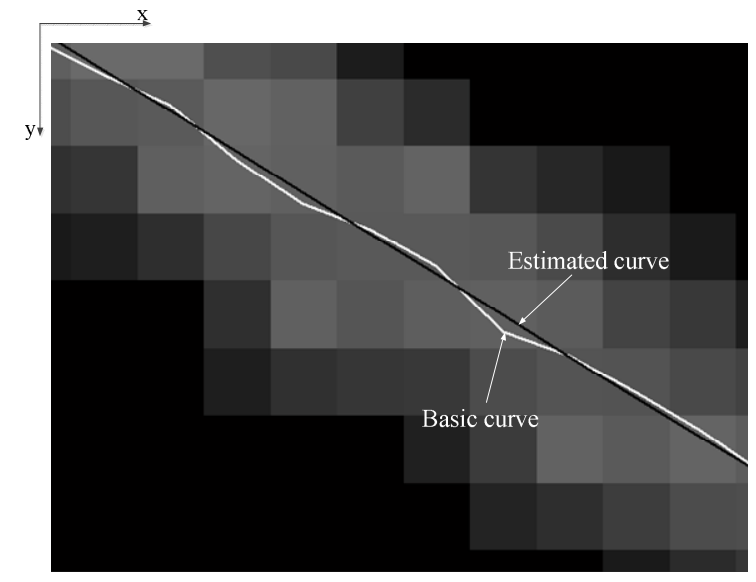

Fig. 6. Estimated curve

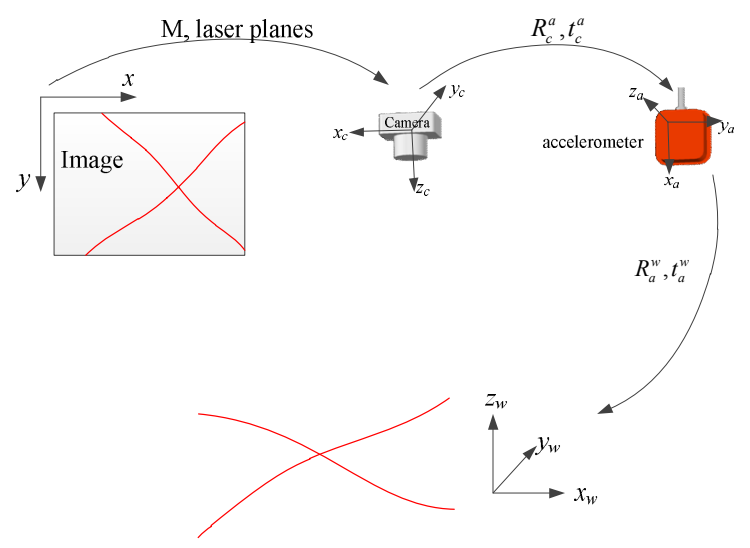

Fig. 7. Coordinate frame conversion 
an accelerometer was used together with a laser-camera system.

The most important factor in estimating golf slope is the vertical which can be represented by the gravitational force. It is assumed that while capturing the golf slope the system is not moving. This assumption guarantees that the output of the accelerometer only contains the gravitational acceleration. Since the $x$ and $y$ direction of the world coordinate frame are not important, the rotation from the accelerometer coordinate frame to the world coordinate frame can be estimated by applying a triad algorithm [15] for the following:

$$
\left[\begin{array}{l}
0 \\
0 \\
1
\end{array}\right]=R_{a}^{w}\left[\begin{array}{l}
y_{a_{x}} \\
y_{a_{y}} \\
y_{a_{z}}
\end{array}\right],\left[\begin{array}{l}
1 \\
0 \\
0
\end{array}\right]=R_{a}^{w}\left[\begin{array}{l}
1 \\
0 \\
0
\end{array}\right],
$$

where $y_{a}=\left[\begin{array}{lll}y_{a_{x}} & y_{a_{y}} & y_{a_{z}}\end{array}\right]^{T}$ is the output of the accelerometer and $R_{a}^{w}$ is the rotation matrix from the accelerometer coordinate frame to the world coordinate frame. Let $R_{c}^{a}$ be the rotation matrix from the camera coordinate frame to the accelerometer coordinate frame. Since the translation between the camera, accelerometer and world coordinate frame is not important, the origins for these three coordinate frames are assumed to be in the same position. For a point $Q_{c}$ in the camera coordinate frame, its coordinates in the world coordinate frame can be express by

$$
Q_{w}=R_{a}^{w} R_{c}^{a} Q_{c}
$$

Therefore, the coordinates of each laser point $Q\left(x_{m}, y_{m}\right)$ in the image is computed in the world coordinate frame as:

$$
Q_{w}^{i}=R_{a}^{w} R_{c}^{a} s T^{-1}\left[\begin{array}{l}
x_{m} \\
y_{m} \\
1
\end{array}\right]=\left[\begin{array}{c}
x_{w}^{i} \\
y_{w}^{i} \\
z_{w}^{i}
\end{array}\right]
$$

where $T$ is the intrinsic matrix obtained from the camera calibration step [10]. Note that $z_{w}^{i}$ shows the height of the point $Q_{w}^{i}$, thus the relationship between the slope and the world coordinate frame can be identified based on $z_{w}^{i}$.

\subsection{Slope surface reconstruction}

Section 4.2 gives two laser stripes of coordinate points which represent the captured slope surface. However, only by looking at these two 3D stripes, it might be difficult for the users to imagine the slope. The objective of this section is to reconstruct the slope surface based on given $3 \mathrm{D}$ coordinate points.
The reconstruction method used in this section was proposed by John R. D'Errico [16]. In [16], the interpolation problem is expressed as a linear algebra problem such as:

$$
A_{p} \mathbf{x}=\mathbf{y}
$$

where the vector $\mathbf{x}$ has $n x \cdot n y$ length ( $n x$ is the number of nodes in the $\mathbf{x}$ direction, and $n y$ is the number of grid nodes in the $\mathbf{y}$ direction). Thus $A_{p}$ has $n_{p}$ rows, corresponding to each data point supplied by the user, and $n x \cdot n y$ columns.

At every node of the surface, interpolation algorithm will try to make the partial derivatives of the surface in neighboring cells to be equal. As the result, we have the linear equation

$$
B_{p} \mathbf{x}=0
$$

where the derivatives are approximated using finite differences of the surface at neighboring nodes.

The combination of (22) and (23) results an optimization problem as follow: find $\mathbf{x}$ to minimize

$$
\left\|A_{p} \mathbf{x}-\mathbf{y}\right\|^{2}+\lambda\left\|B_{p} \mathbf{x}\right\|^{2}
$$

where $\lambda$ is a chosen parameter nominally 1 allows the user to control the relative grid stiffness.

\section{Experiments}

In this section, we verify the accuracy of the proposed system through experiments.

\subsection{Flat slope angle estimation}

In this experiment, the accuracy of flat slope angle estimation using the proposed system was investigated by measuring the angle error between a flat plane and the vertical. In reality, the golf course is almost flat and has a

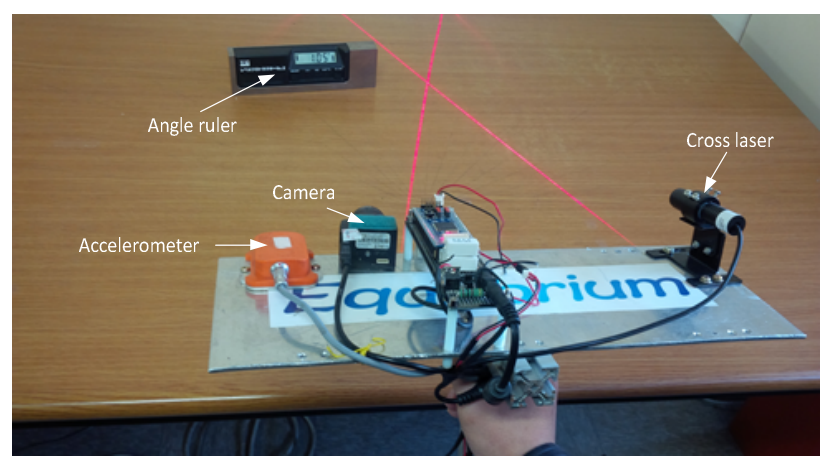

Fig. 8. Slope angle estimation experiment setup 
Table 1. Flat slope angle estimation

\begin{tabular}{|c|c|c|c|c|c|c|c|c|c|}
\hline \multicolumn{10}{|c|}{ True slope angles $(\alpha)$ in degrees } \\
\hline \multicolumn{2}{|c|}{$\alpha=0.55^{\circ}$} & \multicolumn{2}{|c|}{$\alpha=14.10^{\circ}$} & \multicolumn{2}{|c|}{$\alpha=31.55^{\circ}$} & \multicolumn{2}{|c|}{$\alpha=43.05^{\circ}$} & \multicolumn{2}{|c|}{$\alpha=63.25^{\circ}$} \\
\hline \multicolumn{10}{|c|}{ Estimated slope angles $(\hat{\alpha})$ and absolute estimation error $(\tilde{\alpha})$ in degrees } \\
\hline$\hat{\alpha}$ & $\bar{\alpha}$ & $\hat{\alpha}$ & $\bar{\alpha}$ & $\hat{\alpha}$ & $\tilde{\alpha}$ & $\hat{\alpha}$ & $\tilde{\alpha}$ & $\hat{\alpha}$ & $\bar{\alpha}$ \\
\hline 0.620 & 0.070 & 13.898 & 0.201 & 31.542 & 0.008 & 43.008 & 0.042 & 63.376 & 0.126 \\
\hline 0.688 & 0.138 & 13.935 & 0.165 & 31.482 & 0.068 & 43.033 & 0.017 & 63.391 & 0.140 \\
\hline 0.596 & 0.046 & 13.934 & 0.166 & 31.437 & 0.113 & 43.050 & 0.000 & 63.352 & 0.102 \\
\hline 0.626 & 0.076 & 13.978 & 0.122 & 31.446 & 0.103 & 43.118 & 0.068 & 63.342 & 0.092 \\
\hline 0.652 & 0.102 & 13.942 & 0.157 & 31.439 & 0.111 & 43.100 & 0.050 & 63.220 & 0.030 \\
\hline 0.645 & 0.095 & 13.996 & 0.103 & 31.421 & 0.129 & 43.114 & 0.063 & 63.435 & 0.185 \\
\hline 0.664 & 0.114 & 13.917 & 0.182 & 31.466 & 0.084 & 43.117 & 0.066 & 63.418 & 0.168 \\
\hline 0.678 & 0.128 & 13.903 & 0.197 & 31.467 & 0.083 & 43.084 & 0.034 & 63.365 & 0.114 \\
\hline 0.566 & 0.016 & 14.067 & 0.033 & 31.526 & 0.023 & 43.057 & 0.007 & 63.079 & 0.171 \\
\hline 0.579 & 0.029 & 14.005 & 0.095 & 31.543 & 0.007 & 43.072 & 0.022 & 63.149 & 0.101 \\
\hline \multicolumn{10}{|c|}{ Mean estimation error for each slope in degrees } \\
\hline \multicolumn{2}{|c|}{$\tilde{\alpha}=0.081^{\circ}$} & \multicolumn{2}{|c|}{$\tilde{\alpha}=0.143^{\circ}$} & \multicolumn{2}{|c|}{$\tilde{\alpha}=0.073^{\circ}$} & \multicolumn{2}{|c|}{$\tilde{\alpha}=0.037^{\circ}$} & \multicolumn{2}{|c|}{$\tilde{\alpha}=0.123^{\circ}$} \\
\hline \multicolumn{10}{|c|}{ Total mean estimation error in degrees: $\quad \tilde{\alpha}=0.091^{\circ}$} \\
\hline
\end{tabular}

small decent angle that can be approximated as a tilted flat plane. The experiment setup is described in Fig. 8 where the proposed system projects a cross laser line on a flat plane to estimate the flat plane's 3D equation. The angle of the slope can be deduced from the angle formed by the flat plane's normal vector and the vertical vector. The estimated angle is compared with the value obtained from an inclinometer to evaluate the estimation accuracy.

The flat plane was placed in various positions with different angles with respect to the vertical. Projection points from the laser are approximated as a plane in the camera coordinate frame using a least square algorithm [17]. A normal vector computed from the plane's equation is then expressed in the world coordinate frame. This vector makes an $\hat{\alpha}$ angle with the vertical vector that is compared with the ground truth angle $\alpha$ obtained from an inclinometer to find the estimation error $\tilde{\alpha}$ :

$$
\tilde{\alpha}=|\alpha-\hat{\alpha}|
$$

Ten measurements of the slope angle are shown in Table 1. As can be seen from Table 1, the estimation gives a high accuracy with a mean average error of $0.0910^{\circ}$ where the maximum error was $0.201^{\circ}$. In application, the angle of the slope can be computed by

$$
\bar{\alpha}=\frac{\pi}{2}-\hat{\alpha} .
$$

\subsection{Complex Slope Angle Estimation}

This experiment provides a 3D view of a complex slope with respect to the user view. Based on the estimated laser projection curves the slope is depicted with the slope's characteristics such as relative height and angle. In this experiment, a green slope model with $1.2 m \times 1.2 m$ size was

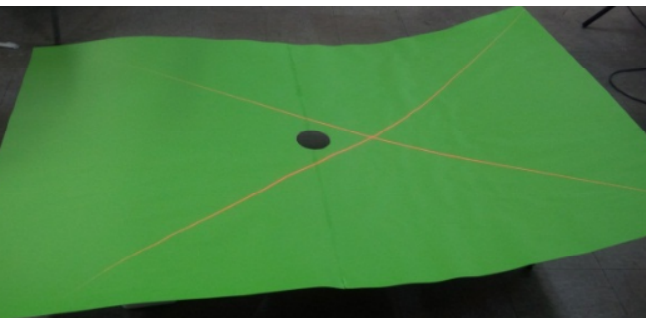

(a) Experiment setup

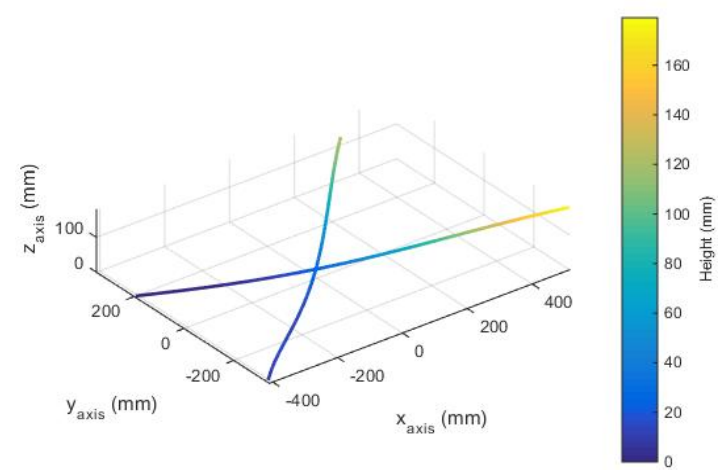

(b) Slope relative height represented by laser stripes

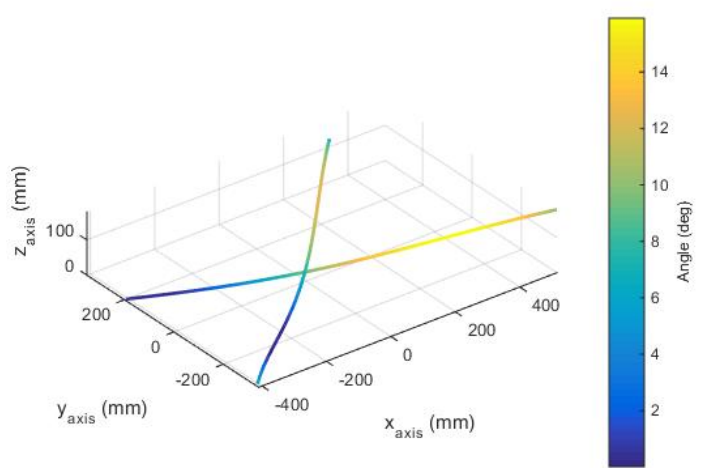

(c) Slope relative angle represented by laser stripes

Fig. 9. Slope angle trend estimation 


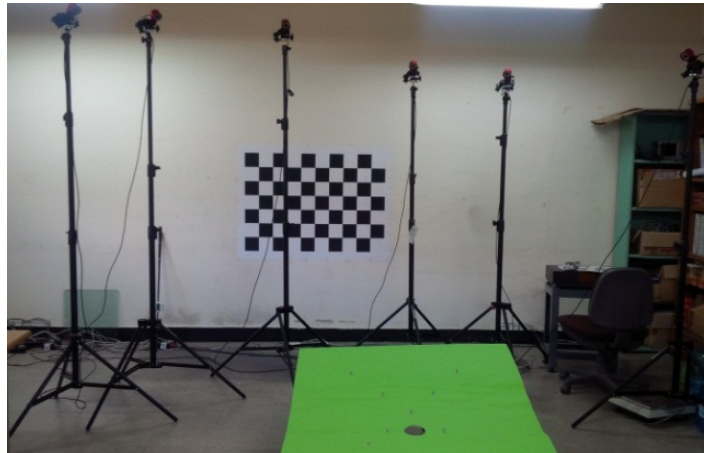

(a) Experiment setup

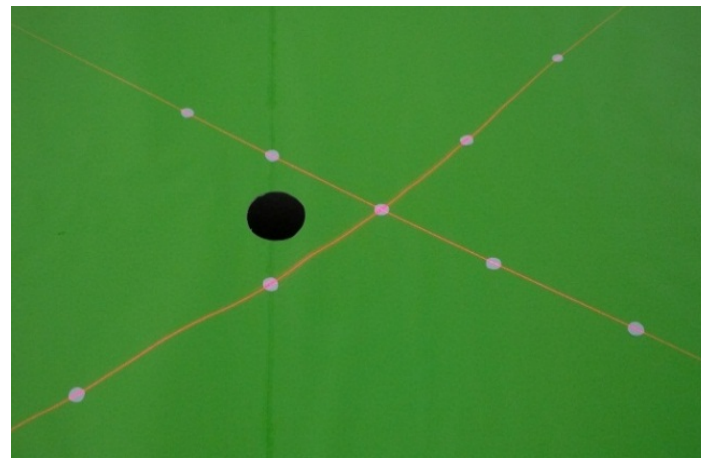

(b) Green slope with markers observed by OptiTra

Fig. 10. Slope angle accuracy verification experiment

Table 2. Marker position estimation errors in different poses

\begin{tabular}{|c|c|c|c|c|c|c|c|c|c|c|}
\hline Marker & 1 & 2 & 3 & 4 & 5 & 6 & 7 & 8 & 9 & RMS \\
\hline \multirow{5}{*}{$\begin{array}{l}\text { Distance } \\
\text { error }(m m)\end{array}$} & 1.08 & 2.74 & 2.44 & 5.13 & 4.59 & 4.31 & 4.69 & 1.53 & 3.72 & 3.63 \\
\hline & 1.93 & 2.62 & 1.10 & 2.58 & 3.82 & 4.81 & 2.48 & 2.76 & 1.63 & 2.84 \\
\hline & 5.77 & 5.73 & 1.58 & 0.97 & 3.97 & 3.22 & 1.64 & 0.59 & 5.09 & 3.72 \\
\hline & 3.80 & 3.75 & 3.72 & 2.55 & 3.51 & 2.41 & 2.18 & 1.58 & 5.00 & 3.32 \\
\hline & 4.99 & 2.92 & 2.34 & 3.67 & 4.47 & 2.66 & 2.94 & 0.86 & 1.54 & 3.18 \\
\hline \multicolumn{10}{|c|}{ Mean } & 3.34 \\
\hline
\end{tabular}

RMS: Root Mean Square of distance error

used as in Fig. 9a.

Fig. $9 \mathrm{~b}$ represents the slope via estimated height while Fig. 9c shows the estimated angle of each point in the laser projections with respect to the world coordinate frame. The color in Fig. 9b changes from purple to yellow respectively with the relative height from the smallest value $(0 \mathrm{~mm})$ to the largest value $(180 \mathrm{~mm})$. Similarly, Fig. 9c illustrates the angle value of each point. The yellow color indicates that at that point the angle of the slope is $16^{\circ}$.

The slope angle is computed based on the tangent of the slope at each point corresponding to the horizontal plane. To reduce computation, the process of defining the slope angle is sketched as following:

- Rotate each laser plane so that its normal vector coincides with the vertical vector. Thus the 3D laser curve becomes $2 \mathrm{D}$ in $z=0$ plane.

- Compute the tangent vector $v$ at each point in 2D.

- Convert the tangent vector $v$ back to 3D in the world coordinate frame.

- Compute the slope angle $\bar{\alpha}$ made by tangent vector and the vertical vector $z_{n}$ :

$$
\bar{\alpha}=\frac{\pi}{2}-\operatorname{acos}\left(\frac{\left(v, z_{n}\right)}{\|v\|\left\|z_{n}\right\|}\right)
$$

The estimated slope accuracy is evaluated using the OptiTrack camera system. The overview of the experiment is described in Fig. 10a where a green slope model is set within the camera system's working field. Projecting the cross-line laser on the slope model, the 3D shape of the slope is computed and expressed in the world coordinate frame using proposed algorithm in Section 4. To verify the

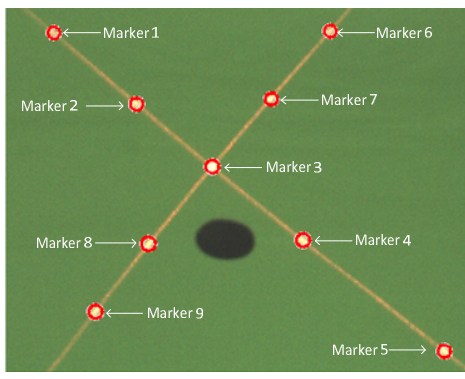

(a) Markers on laser stripes

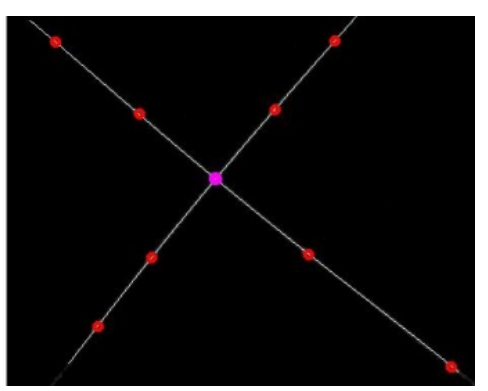

(b) Extracted markers and laser curves

Fig. 11. Markers positioning in camera image

accuracy of the estimation some circle markers are placed on the laser projection stripes so that centers of the markers lie on the stripes. These markers are observed by the camera system to give the ground truth values that will be used to compare with the corresponding points in estimated curves (see Fig. 10b).

The position of the markers in the camera image can be defined by extracting circle shapes as can be seen from Fig. 
11a-b.

Fig. 11a-b shows the coordinates of nine markers in the world coordinate frame as well as the corresponding points estimated from the proposed system. The result is given in Fig. 12, where the proposed system provides an accurate estimation of the slope since the estimated points are close to the true values. The estimation accuracy is also investigated through five times measuring the slope in different poses of the laser system. The result is given in Table 2. Table 2 shows the distance error of each point corresponding to the marker position in millimeter. The root mean square of the distance error is used as an evaluating criterion. The table indicates that our proposed system provides an accurate estimation of the golf slope with the mean distance error of $3.34 \mathrm{~mm}$.

To provide a visual expression for the user, the surface of the slope is reconstructed based on the estimated laser points. Fig. 13 shows the reconstruction result of the slope. The height of the slope is represented by color for an easy imagination. By looking at the reconstructed slope, the user can easily understand the slope and have a better prediction of the golf ball trajectory.

In this experiment, the reconstructed surface was built based on 831 3D laser points within 0.072 seconds in a Core i5 3.00GHz, 8GB RAM PC. This speed implies that

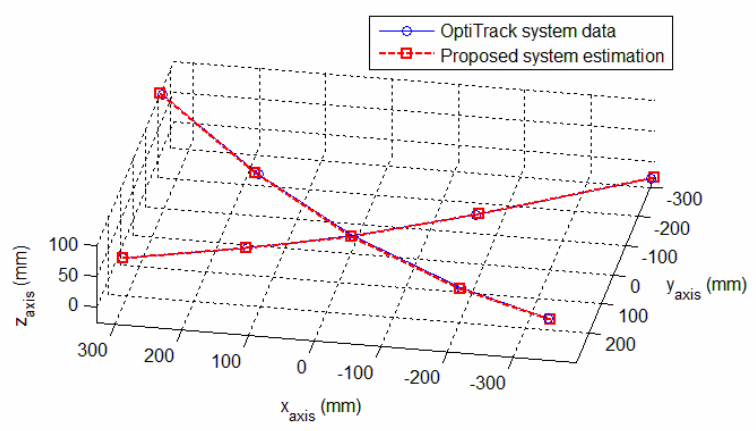

Fig. 12. Estimated and true slope coordinate points
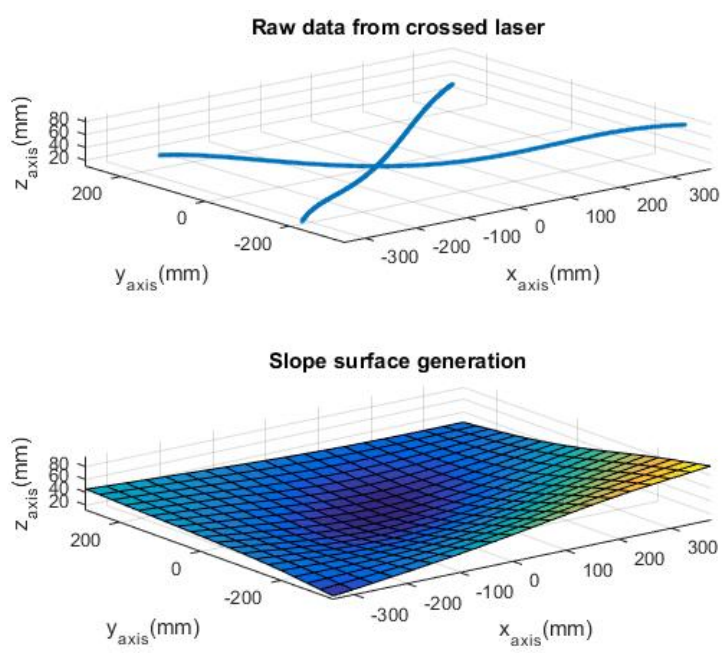

Fig. 13. Slope surface reconstruction

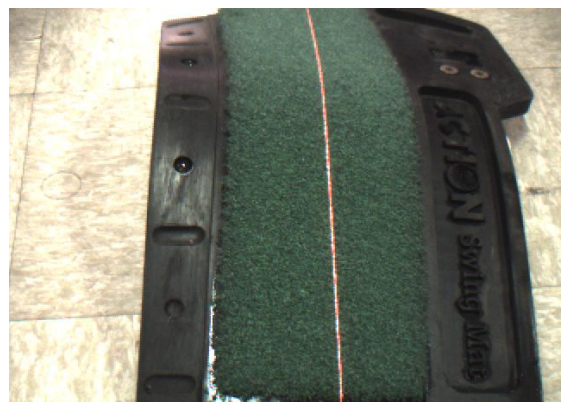

(a) Golf hitting mat with laser stripe

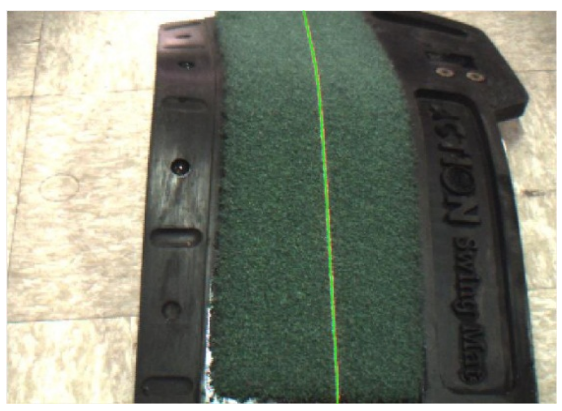

(b) Golf hitting mat with extracted laser points

Fig. 14. Slope surface reconstruction

the proposed method can be applied in a smartphone for a quick surface generation for golfers while playing.

In the next experiment, we examined the effect of the grass on the green surface on the slope estimation quality. In reality, there are grasses in the golf green, however, is very short (from 2.25 to $4 \mathrm{~mm}$ [18]) and dense. In this experiment, we check the feasibility of extracting laser stripes in an artificial grass environment. The experimental object was a golf hitting mat with $5 \mathrm{~mm}$ height grass. The experimental process was the same as it is proposed in Section 4 with image subtraction to obtain laser stripe from captured images.

The result in Fig. 14 shows that, the system can extract the laser stripe successfully in grass slope. After this step, the slope is estimated totally the same as it is mentioned in Section 5.

\section{Conclusion}

The paper proposed a system consisting of an accelerometer, a cross structured light and a camera to estimate the surface structure of the golf green slope. In order to estimate the golf slope, a method to detect laser planes as well as laser projection curves was proposed. Laser projections are separated by utilizing the fact that the intersection of two laser planes is fixed with respect to the camera and image coordinate frame. After the projections are distinguished, the laser planes' equations are computed using an optimization problem. Hence, all the intersection 
point coordinates of the laser planes and the slope can be computed with respect to the world coordinate frame. Based on these points, the slope surface is reconstructed using an interpolation method.

To verify the feasibility of the proposed system some experiments have been done. The result shows that our system can work with a high accuracy of $0.1^{\circ}$ in flat slope angle error and $3.34 \mathrm{~mm}$ for mean distance error in complex slope. In addition, an experiment on an artificial grass mat verified that the system still can extract the laser points from the grass surface.

Using the system, the golf slope is presented in $3 \mathrm{D}$ that provides an insight view of the slope's characteristics for the players. Furthermore, the system is light, simple and cheap, which can be easily applied in a smart phone where a high resolution camera and an accelerometer are available. By simply attaching a laser light the smart phone can become our proposed system which is very convenient for players to use in the golf field.

\section{Acknowledgements}

This work was supported by the 2014 Research Fund of University of Ulsan.

\section{References}

[1] L. Zhang, Q. Ye, W. Yang, and J. Jiao, "Robust Weld Line Detection and Tracking via Spatial-Temporal Cascaded Hidden Markov Models and Cross Structured Light," IEEE Transaction on Instrumentation and Measurement, vol. 6, pp. 742-752, 2014.

[2] Tom Botterill, Steven Mills, and Richard Green, "Design and calibration of a hybrid computer vision and structured light 3D imaging system," presented at the International Conference on Automation, Robotics and Applications, Wellington, New Zealand, 2011.

[3] Din-Chang Tseng and Zen Chen, "Computing Location and Orientation of Polyhedral Surfaces Using a Laser-Based Vision System," IEEE Transaction on Robotics and Automation, vol. 7, 1991.

[4] Ben Thomas and A. Jothilingam, "Object Identification Based on Structured Light Reconstruction and CAD Based Matching," International Journal of Innovative Research in Science, Engineering and Technology, vol. 3, 2014.

[5] R.J Valkenburg and A.M. Mclvor, "Accurate 3D measurement using a structured light system," Image and Vision Computing, vol. 16, pp. 99-100, 1998.

[6] W.Van Lier, J.Van Der Kamp, and G.J.P. Savelsbergh, "Gaze in golf putting: effects of slope," International Journal of Sport Psychology, vol. 41, pp. 160-176, 2010 .
[7] Heiko Hecht, Dennis Shaffer, Behrang Keshavarz, and Mariagrace Flint, "Slope estimation and viewing distance of the observer," Attention Perception \& Psychophysics, vol. 76, pp. 1729-1738, 2014.

[8] Inclinometers: Mechanical \& Electronic. Available: $\mathrm{http}: / /$ riekerinc.com/Inclinometers.htm

[9] Kenichi Kanatani, Statistical Optimization for Geometric Computation: Dover Publications, 1996.

[10] Gary Bradski and Adrian Kaehler, Learning OpenCV: O’Reilly Media, 2011.

[11] Zhengyou Zhang, "A Flexible New Technique for Camera Calibration," IEEE Transaction on Pattern Analysis and Machine Intelligence, vol. 22, pp. 13301334, 2000.

[12] Richard Hartley and Andrew Zisserman, Multiple View Geometry in Computer Vision. Cambridge: Cambridge University Press, 2003.

[13] Jorge Lobo and Jorge Dias, "Relative Pose Calibration Between Visual and Inertial Sensors," The International Journal of Robotics Research, vol. 26, pp. 561-575, 2007.

[14] Daesik Kim, Seongsoo Lee, Hyunwoo Kim, and Sukhan Lee, "Wide-Angle Laser Structured Light System Calibration with a Planar Object," presented at the International Conference on Control, Automation and Systems, KINTEX, Gyeongki-do, Korea, 2010.

[15] Harold D. Black, "A passive system for determining the attitude of a satellite," AIAA Journal, vol. 2, pp. 1350-1351, 1964.

[16] J. R. D'Errico. (2006, August 26). Surface Fitting Uisng Gridfit. Available: http://www.mathworks.com/ matlabcentral/fileexchange/8998-surface-fittingusing-gridfit

[17] Edwin K. P. Chong and Stanislaw H. Zak, An Introduction to Optimization. New York, The USA: John Wiley \& Sons, inc., 2001.

[18] A. Dunsmuir. (2011). Height of cut: Tread with caution when comparing golf courses. Available: http://www.golfclubmanagement.net/2011/12/heightof-cut-tread-with-caution-when-comparing-two-golfcourses/

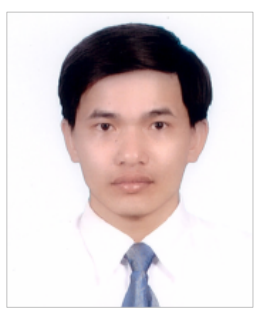

Duy Duong Pham He received his B.S. degree of Automation from Department of Electrical Engineering at Danang University of Technology The University of Nanang, Vietnam in 2010. From 2011 he works as a lecturer at Danang College of Technology - The University of Nanang. He is currently pursuing the Master degree at University of Ulsan. His research interests include mobile robot control, motion tracking and personal navigation. 


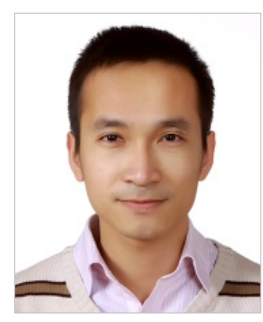

Quoc Khanh Dang $\mathrm{He}$ received his B.S. degree of Automation from Department of Electrical Engineering at Hanoi University of Science and Technology, Vietnam in 2009. In 2012, he received his M.S. degree from School of Electrical Engineering at University of Ulsan, Korea. He is currently pursuing the PhD degree at University of Ulsan. His research interests include mobile robot control, motion tracking and personal navigation.

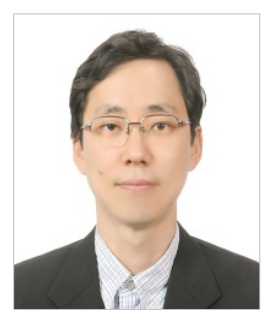

Young-Soo Suh He received his B.S. and M.S. degrees from the Department of Control and Instrumentation Engineering at Seoul National University, Korea in 1990 and 1992, and his Ph.D. degree from the Department of Mathematical at Engineering and Information Physics at the University of Tokyo, Japan in 1997. He is currently a professor in the Department of Electrical Engineering, University of Ulsan, Korea. His research interests include networked control systems and attitude estimation and control, and personal navigation systems. 